Rivista di Storia delle Religioni

$10 \mid 2016$

Varia

\title{
Schicksalsvorstellungen im Späten Ägypten
}

Conceptions of Fate in Late Egypt

\section{Joachim Friedrich Quack}

\section{OpenEdition}

\section{Journals}

\section{Edizione digitale}

URL: http://journals.openedition.org/mythos/454

DOI: $10.4000 /$ mythos. 454

ISSN: 2037-7746

\section{Editore}

Salvatore Sciascia Editore

\section{Edizione cartacea}

Data di pubblicazione: 1 décembre 2016

Paginazione: 43-60

ISBN: 978-88-8241-476-4

ISSN: $1972-2516$

\section{Notizia bibliografica digitale}

Joachim Friedrich Quack, "Schicksalsvorstellungen im Späten Ägypten », Mythos [En ligne], 10 | 2016, mis en ligne le 24 septembre 2019, consulté le 28 septembre 2019. URL : http://

journals.openedition.org/mythos/454 ; DOI : 10.4000/mythos.454 


\section{Schicksalsvorstellungen im Späten Ägypten}

\section{Joachim Friedrich Quack}

\section{Zusammenfassung}

Es gibt zwei ganz verschiedene mögliche Herangehensweisen, was die Unveränderlichkeit des Schicksals betrifft. Die eine ist, es als unausweichlich zu betrachten, so daß jede Vorhersage nichts weiter kann, als die Menschen darauf vorzubereiten, was sie erwartet. Die andere besteht darin, durch Gebete und Ritual die Götter zu einer Änderung zu bewegen, insbesondere drohende Schäden zu verhindern. In diesem Artikel werden divinatorische, literarische und religiöse Texte aus dem späten Ägypten daraufhin untersucht, wie die Ägypter in dieser Zeit das Schicksal verstanden haben. Die Quellen zeigen deutlich, daß man von den Göttern meinte, sie ständen oberhalb des Schicksals, und es sei möglich, schlechte Vorhersagen abzuwenden.

\begin{abstract}
There are two quite different approaches possible concerning the unchangeability of destiny. One is to see it as ineluctable, so that any prediction can do no more good than to prepare the man for what is coming. The other one is that prayers or rituals can induce the gods to change it, especially to repeal bad outcomes. In this article, divinatory, literary and religious texts from Late Egypt are studied in order to how the Egyptians at this time vied destiny. The sources demonstrate clearly that the gods were considered to stand above destiny, and that it was thought possible to avert bad predictions.
\end{abstract}

\section{Schlüsselworte}

Ägypten, Spätzeit • Divination • Literatur • religiöse Texte • Schicksal

\section{Keywords}

Egypt, Late Period • divination - literature • religious texts • destiny

$\mathrm{n}$ den Anfang meiner Ausführungen möchte ich zwei sehr divergente mögliche Zu-
gangsweisen hinsichtlich der Relevanz und Unabwendbarkeit des Schicksals stellen.
Beide wähle ich, um hinsichtlich des Alten Ägyptens nichts zu präjudizieren, aus anderen antiken Kulturen aus.

Die eine möchte ich als das philosophische (speziell stoische) Paradigma bezeichnen. In ihm ist das Schicksal unabwendbar. Dies hat entsprechende Folgen auch für die Relevanz der Divination. Was bringt die Vorhersage der Zukunft, wenn das Schicksal ohnehin feststeht? ${ }^{1}$ Cicero stellt in seiner Rolle als divinationskritischer Gesprächspartner in seinem Dialog De divinatione die Frage, wozu es für den Menschen überhaupt gut sei, die Zukunft zu kennen, wenn er sie doch nicht ändern könne (II, 20-25; 105). Diese Position erkennt die Prämisse an, dass das Schicksal, sofern es überhaupt im Voraus erkennbar ist, als etwas Unveränderliches dasteht, nicht jedoch noch abgewendet werden kann. Diese Position scheint in der weiteren Geistesgeschichte noch mehrfach aufgegriffen worden zu sein. So findet sie sich bei Seneca, Quaestiones naturales II, XXXVf., der betont, das Schicksal sei nicht durch Gebete zu beeinflussen. Allerdings räumt Seneca bereits die Option ein, dass die Götter sich durch 
Opfer besänftigen liessen, sofern es dem Empfänger der Vorzeichen vorbestimmt sei, zu opfern (Quaestiones naturales II, XXXVI-XXXVIII). Hier zeigt sich de facto das Problem, eine strikt deterministische Auffassung konsequent durchzuhalten.

Erneut erscheint eine derartige Diskussion bei Lukian, Astrologia, 28f., wo daran erneut die Frage geknüpft wird, ob Zukunftsvorhersage gut und nützlich ist. Er argumentiert zum Lob der Astrologie, es sei für den Menschen gut zu wissen, was komme, damit er sich innerlich darauf vorbereiten und sich so im Voraus auf künftiges Glück freuen, Schicksalsschläge dagegen leichter mit Fassung tragen könne. Etwa zur selben Zeit argumentiert auch Ptolemaios, die Astrologie sei auch dann nötig, wenn die Ereignisse zwangsläufig eintreten, weil man sie dann mit Ruhe und Gelassenheit aufnehmen könne (Tetrabiblos I, 3). Dabei rechnet er allerdings damit, dass nicht alle Ereignisse unabänderlich sind.

Man könnte natürlich, wenn man den Determinismus noch konsequenter anlegt, Lukians (und bedingt Ptolemaios') Position dahingehend kritisch hinterfragen, ob der Mensch denn überhaupt die freie Möglichkeit habe, sich für zukünftige Schicksalsschläge zu wappnen oder nicht auch diese innere Haltung mit vorherbestimmt sei, aber das möchte ich nicht vertiefen. Zudem muß bedacht werden, dass zumindest Lukian seine vorgebliche Meinung eventuell durchaus nicht ernst nimmt, sondern vielmehr die Position der Astrologie-Anhänger ad absurdum führen will, indem er sie auf die Spitze treibt, wie er ja auch in Jupiter confutatus, 12-15 einen Kyniker gegen Zeus argumentieren lässt, Schicksalsankündigungen würden zu nichts gut sein, da man dem Verderben doch nicht entrinnen könne - und damit bleibt für die Götter kein Bereich mehr, in dem sie jenseits der Spindel der Notwendigkeit etwas eigenes beitragen können.

Das zweite denkbare Paradigma möchte ich als das Namburbi-Schema bezeichnen. Der Begriff kommt aus dem Alten Orient und bezeichnet zunächst in der sumerischen Sprache, dann als Lehnwort auch im Akkadischen Rituale, mit denen man bevorstehendes Unheil abwenden will. Diese Verfahren wurden von Stefan Maul mit dem treffenden Begriff „Zukunftsbewältigung" im Detail analysiert. ${ }^{2}$ Sie stehen im Rahmen einer intensiven Beobachtung von Vorzeichen, bei der man den Auftraggebern (besonders dem Königshof) Meldung von drohenden Schicksalsschlägen erteilt und gleichzeitig Kompetenz für Rituale hat, mit denen spezifische oder allgemeine Unheilsdrohungen abgewendet werden können. Die Verfahren als solche richten sich an die Götter, denen somit die Kompetenz zugeschrieben wird, das einmal verhängte Schicksal auch wieder aufzuheben. Im Rahmen einer solchen Sicht ist die Divination eindeutig eine gute und wichtige Technik, da sie drohendes Unheil dann anzeigt, wenn man es durch korrektes Verhalten noch abwenden kann.

Es wird wohl nicht zu sehr überraschen, wenn ich von vornherein feststelle, dass die ägyptische Haltung gegenüber dem Schicksal tendenziell deutlich dem zweiten Paradigma zuneigt, dass Schicksal nicht unabänderlich ist. ${ }^{3}$ Die konkreten Ausprägungen lohnt es aber im Detail zu untersuchen. Hierbei möchte ich auf drei sehr verschiedene Bereiche fokussieren, um ein insgesamt möglichst abgerundetes und facettenreiches Bild zu erhalten.

Als erstes möchte ich die Haltung der divinatorischen Praxis untersuchen. So sehr die frühere Forschung irrig behauptet hat, das Alte Ägypten sei keine divinatorische Kultur gewesen, ${ }^{4}$

2 Maul 1994.

3 Vgl. hinsichtlich der Frage des Schicksals in Ägypten besonders Morenz, MüLler 1960.

4 Assmann 1996, 233f.; ders., 1999, 15-19; ders., 2005, 113. 
so sehr sind doch wenigstens aus dem späten Ägypten erhebliche Mengen sowohl an theoretischen Handbüchern als auch an Hinterlassenschaften in Form praktischer Anfragen erhalten. ${ }^{5}$ Die realen Anfragen ${ }^{6}$ zeigen sicher an, dass die dahinter stehenden Personen es nützlich empfunden haben, Wissen über die Zukunft zu erhalten, geben aber meist keine klaren Anzeichen dafür, ob sie schlechte Vorhersagen dann noch abwenden oder sich nur innerlich stählen wollten, um sie gefasst aufzunehmen. Zudem handelt es sich meist um Fragen darüber, wie man sich selbst verhalten soll, weniger dagegen darüber, was einem zustösst. Aufschlussreicher sind hier die Herangehensweisen der theoretischen Traktate. Bei ihnen möchte ich wiederum drei verschiedene Gattungen unterscheiden, nämlich terrestrische Omina, Traumomina und astrologische Traktate.

Unter den terrestrischen Omina ist der Hauptzeuge ein noch weitgehend unpubliziertes grosses Handbuch. ${ }^{7}$ Es enthält vorwiegend Vorhersagen anhand des Verhaltens von bzw. des Kontaktes mit Tieren, daneben auch Brennverhalten von Lampen. Insgesamt erscheint es dem mesopotamischen Traktat Šumma älum nicht unähnlich. Dass die positiven Vorhersagen des Textes nicht vorbehaltsbewehrt sind, sondern einfach wie eine Gewissheit angekündigt werden, überrascht kaum. Dagegen wird bei unangenehmen Prognosen, insbesondere solchen, die reale Gefahr für Leib und Leben ankündigen, meist mit Optionen der Rettung gearbeitet, während die direkte und ungemilderte Todesankündigung ausgesprochen selten ist. Teilweise begnügt man sich mit einer simplen Warnung; so etwa $h r h \underline{h} r . r=f r-c ̧ b^{3} y^{c} b y^{r} \breve{c}^{\mathfrak{c}} t h g^{3}$, ,achte auf ihn wegen Infektion oder Haft!“ oder my ir $\underline{i}=f h r h \underline{r} r . r=f r-c ̧ b 3$ 3sy „Möge er sich vor Verlust hüten!“. Als weitergehende Schutzmittel können einerseits Schutzzauber und Amulette verwendet werden, so $m y{ }^{C_{s}}=w n=f s \underline{h}$ ) $m y$ y $m=w n=f s \jmath$. $w$, möge man für ihn Schriften rezitieren

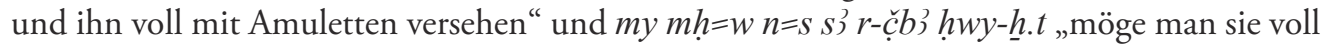
mit Amuletten gegen Fehlgeburt versehen“"

Gebräuchlicher ist aber das Flehen zu den zuständigen Göttern, so $i w=f(r) \operatorname{sbh} n$ p ’ nčr $i w=f$

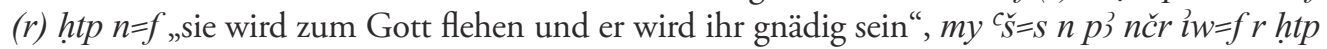
$n=s$,möge sie zum Gott rufen und er wird ihr gnädig sein“, $m y$ ir $r i=f c_{S}^{\check{S}}$... „, möge er flehen ...", $m y$ ir $i=f / s$ sbh „möge er/sie flehen“; fallweise mit Zusatz „zum Gott“; ausserdem noch „er wird

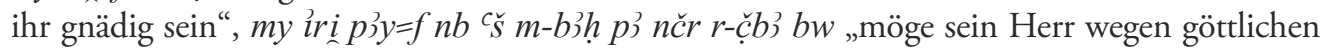
Zorns vor dem Gott flehen “. Konkrete Opfer erwähnt werden in $m y{ }^{c_{s}}=s m-b \grave{h} h$ p ’ nčr $n-r n=f$ (n) hnke krr „möge sie vor dem besagten Gott rufen mit Bier und Brandopfer“. Ähnlich findet

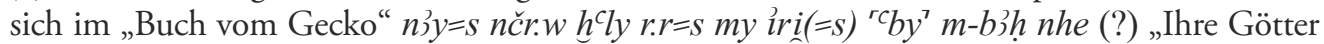
zürnen ihr; möge sie vor ... ein Opfer darbringen. " pBerlin 15680, $\mathrm{x}+2$, 5; my î $\mathrm{r} i=s$ gyl $n$. im $=s$ (n) p) nčr ,möge sie sich dem Gott anvertrauen“ pBerlin 15680, $\mathrm{x}+2,22$ und 29; n3y=s nčr.w gmi $n=s$ lwh my iri=w [gl]l $m-b 3 h=w i w=w(r)$ hri ,ihre Götter finden an ihr Tadel; möge man vor ihnen ein Opfer darbringen, und sie werden besänftigt sein “ pBerlin 15680, x+2, $27 .{ }^{8}$

5 Einen Querschnitt der späten Quellen gebe ich in Übersetzung in QuACK 2008; einen (inzwischen schon wieder deutlich vermehrbaren) Überblick über ägyptische Divination allgemein bietet von Lieven 1999.

6 Neueste Materialzusammenstellung in NAETHER 2010.

7 Die Handschriftenfragmente, an denen ich seit Jahren arbeite, befinden sich in Wien, Berlin, London, Oxford, Aberdeen und Brooklyn; nur das letzte Fragment ist in Hughes 2005, 13, Taf. 16 schon zugänglich gemacht (allerdings nicht bearbeitet) worden. Ein Vorbericht in QUACK 2006, 175-179; QUACK 20 Io. Da die endgültige Numerierung der Fragmente, von denen teilweise solche direkt joinen, die sich heute in verschiedenen Sammlungen befinden, noch nicht feststeht, verzichte ich hier auf Stellenangaben. Für einen verwandten Text (pBerlin 15680) s. ZAUZICH 2012.

8 Meine Lesung weicht hier von ZAUZICH 2012, 358 u. 364 ab. 
Dabei sind, soweit feststellbar, die zuständigen Götter diejenigen, die mit dem Omenanzeiger enger verbunden sind, konkret Bastet mit einem wohl katzenartigen Tier lexikalisch nicht ganz klarer Natur und Astarte mit dem Pferd. Bemerkenswert ist, mit welcher Häufigkeit schon in der Apodosis selbst die göttliche Gnade zugesichert wird. ${ }^{9}$ Zusicherung gött-

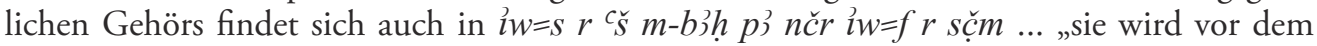
Gott rufen und er wird [ihre Stimme(?)] hören“. In ähnlicher Weise kann auch Befreiung von

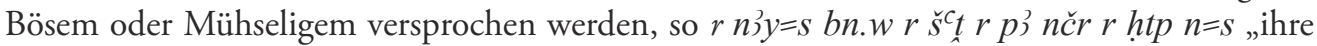
Übel werden beseitigt werden und der Gott wird ihr gnädig sein“, $r n 3 y=f \underline{h} s y[..] \underline{h}$, seine

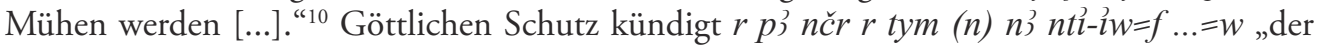

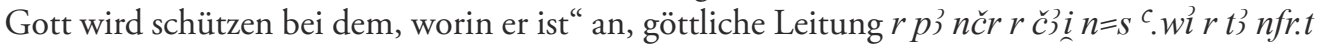
„der Gott wird sie zum Guten leiten“. Die vergleichsweise positive, hoffnungsvolle Tendenz des betreffenden Textes ${ }^{11}$ wird noch umso deutlicher, wenn man berücksichtigt, dass in vielen Fällen zwar negative Ereignisse angekündigt, ihre Folgen aber letztlich als nicht zu gravierend angegeben werden. Von den Krankheitsankündigungen verheissen [iw] $=s i r i y^{c} b y i w=s r i r i$ $b n r$, ,sie wird Infektion erleiden, aber entrinnen“ und $i w=f r i r i y^{c} b y{ }^{r} i w=f r i \hat{i} i^{\top} b n r$,Er wird sich infizieren, aber entrinnen " direkt das Entrinnen, [liw $]^{r}=f^{\prime}[r]$ ir i $i y^{c} b y$ r p ${ }^{\prime} n \check{c} r r$ htp ${ }^{r} n=f^{\prime}$ „Er [wird] sich infizieren, aber der Gott wird ihm gnädig sein“ verspricht Gnade des Gottes. Die Warnung vor Verleumdung $i w=w r h w y{ }^{r} T^{c} r . h r=f^{\prime} n m t . t i w=f(r) i r i \underline{b} b n r$,Man wird ihn in einer Sache verleumden, aber er wird entrinnen“ verspricht ebenfalls das Entrinnen. Eine ungewöhnlich negative Aussicht bietet das „Buch des Gecko“, in dem es einmal heißt $p$ ) nčr

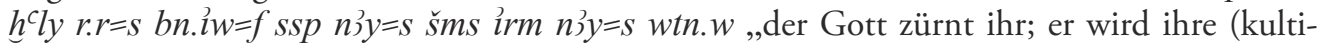
schen) Dienste und ihre Trankopfer nicht annehmen" pBerlin 15680, x+3, $25 \mathrm{f}$.

Man sollte allerdings bemerken, dass all diese Hinweise auf mögliche Gnade sich vorrangig zeigen, wo die Situation als besonders ernst angesehen wird. Bei harmloseren Fällen, in denen es nur um finanzielle Verluste geht, fehlen sie normalerweise.

Während meist das Flehen nur für konkrete einzelne Prognosen angeraten wird, gibt es in zwei Fällen eine Sondersektion. Zum Abschluss des Abschnittes über Vorzeichen von Eulen

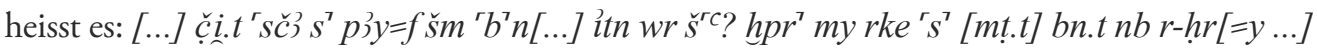
„[Zauber zum] Beseitigen ihres schlechten Omens. [...] grosse Sonne, die zuerst entstand, möge jede üble Sache vor [mir] aufhören.“. Eine ähnliche Passage findet sich auch zum Abschluss der Katzenomina. Hier ist offenbar das Tier als Omenanzeiger so sehr negativ konnotiert, dass man einen wenigstens kurzen Rezitationstext als festen Bestandteil in das divinatorische Handbuch integriert hat. Dies ähnelt übrigens der Situation in Mesopotamien, wo kurze Namburbi-Rituale auch innerhalb der divinatorischen Traktate selbst auftreten können. ${ }^{12}$

Ein fragmentarischer weiterer Papyrus, den ich ebenfalls als Handbuch der Tieromina betrachten würde, zeigt ein ähnliches Bild. ${ }^{13}$ Dort erhalten ist etwa $r n j y=f n c ̌ r . w r$ htp $n=f$,,seine

9 Dabei erlaubt die sprachliche Stilisierung nur die Wiedergabe als echte Zusicherung; für „möge er zum Gott flehen, dass er ihm gnädig sei“ würde im Demotischen wohl der Konjunktiv verwendet werden.

10 Ebenso und ebenfalls unvollständig erhalten im Traumbuchtext pBerlin 8769 A 3, 7.

11 Auch sonst scheint in Ägypten kein fatalistischer Schicksalsglaube zu herrschen, sondern die Vorstellung, sein Schicksal im Zweifelsfall aufbessern zu können, s. von LiEven 1999, $115 f$.

12 Maul 1994, 11 u. 163-165.

13 pKairo 50138 und 50139, publiziert bei SPIEGELBERG 1932, 98-101, Taf. 59 (danach Spiegelbergs Lesung gelegentlich zu berichtigen). Dass es sich um Tieromina, nicht um Traumdeutung handelt, zeigen noch erhaltene Endpartien der Protasen; es könnte in einem Bereich sogar eine freie Parallele zu der Haupthandschrift der Tieromina geben. 


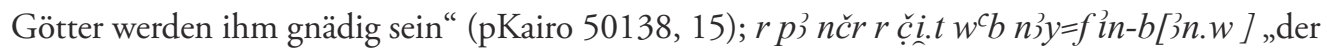
Gott wird seine Übel reinigen“ (pKairo 50138, 19). Auch hier sind konkrete Opfer erwähnt, so

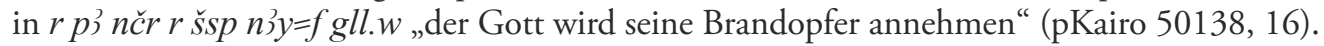

Auf einem relativ ähnlichen Stand liegt auch die Traumdeutung. ${ }^{14} \mathrm{Hier}$ ist allerdings das demotische Material so trümmerhaft erhalten, dass nur bedingt Aussagen möglich sind. Es gibt teilweise durchaus ungeminderte schlechte Ankündigungen, so "Sie wird bald sterben." (pCarlsberg 13 b, 2, 21). In derselben Handschrift heisst es allerdings auch $i w=s r \operatorname{sbh} n b w{ }^{c}$ ) $i w=w[r h t p n=s]$,sie wird wegen eines grossen Frevels flehen und man wird ihr [gnädig sein]“ (pCarlsberg 13, b, 2, 20). Andere Papyri bieten ähnliche Angaben, so $i w=w$ (r) gmi $n=f b w$ iw $=f r h t p n=f$,man wird göttlichen Zorn an ihm finden, er (der Gott?) wird ihm gnädig sein“ (pBerlin 8769, B 2, 15); ; $\left.^{1}\right]^{r} w=w^{7} r$ shm njy=s in-bin.w „man wird ihre Übel vergessen“ (pCarlsberg 14, e, 7); r t’’y=f špšy.t $r$ htp $n=f$,,seine Glücksgöttin wird ihm gnädig sein“ (pJena 1209, 5); $r$ p ’ nčr $r$ htp $n=f$, der G ott wird ihm gnädig sein“ (pJena 1209, 10); [.. r] htp $n=f$ „[... wird] ihm gnädig sein“" (pCarlsberg 13, a, 2, 14).

Ein sehr fragmentarischer Papyrus, dessen Einordnung als Traumbuch oder terrestrische

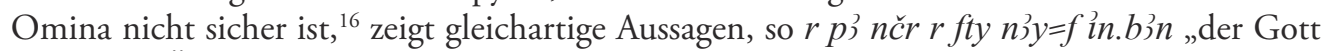
wird seine Übel wegwischen“ (pKairo 50141, 1, x+8); r p 3 nčr $r$ htp $n=f$, der Gott wird ihm gnädig sein" (pKairo 50141, 1, x+7).

Hinweisen möchte ich auch auf einen in der Deutung nicht ganz einfachen Text, bei dem es sich mutmasslich um ein Verfahren in der Art eines Würfelorakels handelt. ${ }^{17}$ Dort heisst es etwa in einer Sektion: „Die Frage, welche Isis, die grosse Göttin, stellte, indem sie sagte: „Werde ich meinen Sohn vor dem Schicksalsgott, Amun, Ptah und dem Sonnengott, den grossen Göttern, empfangen? Werden sie ihm das Diadem geben?“ Der Stein sagte ihr: „Die Frage der Kinder des Königs - Der Schicksalsgott zürnte zunächst gegen sie. Man nahm ihr Herrschaftsamt. Sie flehten vor [ihnen], man war ihnen gnädig, man gab es ihnen. Dies ist die Frage: Man hat ihm sein Haus des Lebens genommen, man wird es ihm wieder geben. Wer im Leid draussen ist, wird kommen, indem er sich freut. Wer in Haft ist, von dem wird man ablassen. Wer krank ist, wird leben. Du sollst zu Amun, dem grossen Gott rufen, er wird deine Stimme erhören. Der grosse Gott(?), rufe ihn an! Er wird es bei dir eintreffen lassen, er wird dir den Schutz des Vorzeichens bereiten, <es> wird rasch zu dir kommen." (pWien D 12006, 3, 6-11).

Das Proprium dieses Textes besteht darin, dass er nicht einfach Vorhersagen für die Zukunft bietet, sondern auf bereits eingetretene Präzedenzfälle verweist, welche die Deutung

14 Wichtige Textpublikationen 1942; ZauzICH 1980; weiteres Material wird von Luigi Prada zur Publikation vorbereitet, s. vorerst Prada 2012a; idem, 2012b; idem, 2013.

15 Vgl. auch pKairo 50139, $\mathrm{x}+4$, wo nur [... sb]h $m$-b $3 \grave{h}$ und $h t p$ klar lesbar sind.

16 pKairo 50141, publiziert bei SpIEgelberg 1932, 103, Taf. 59 (auf der Tafel irrig als 50140 beschriftet).

17 Stadler 2004 (mit Deutung als Divination mit Hilfe eines in Trance versetzten Kindes); s. dazu die Rezensionen von Quack 2005; Winkler 2007; Devauchelle 2008; Richter 2008; Dieleman 2009; Laisney 2010; Übersetzung der besser erhaltenen Passagen in QUACK 2008, 362-367. Für wesentliche Strukturfragen des Textes s. weiter NAETHER 2010, 333-336; neuester Versuch einer Verteidigung seiner ursprünglichen Ansätze bei STADLER 2012, 165-177. Es sollte betont werden, dass für das Schlüsselwort $c l$ die Übersetzung als „Stein“ die einzig philologisch mögliche ist und der von STADLER 2012, 174 als Ausweg versuchte Vorschlag, auch wenn das Steindeterminativ vorläge (und es liegt m.E. sicher vor), könne es sich um eine unetymologische Schreibung handeln, nicht beachtet, dass unetymologische Schreibungen auf grosser Ähnlichkeit oder Identität der Aussprache basieren, eine solche zwischen „Stein“ (kopt. al) und „Kind“ (kopt. alū) aber nicht gegeben ist. 
angeben. Demnach ist in einer ähnlichen Lage schon einmal zur Gottheit gefleht worden, und entsprechend wird auch in der aktuellen Situation Flehen nützlich sein.

Die Astrologie erscheint dagegen als der vergleichsweise deterministischste Bereich der Divination. ${ }^{18}$ In den Traktaten wird normalerweise eine Verkündigung für ein ganzes Leben gegeben, und in ihnen selbst gibt es keine eingestreuten Ritualtechniken, mit denen man sie ändern kann. Ein Textbeispiel ist etwa: „Wer geboren wurde, während Venus im Haus des Dämons war, wird wirr gehen mit einer Ehefrau. Man wird ihn nicht auf eine Ehefrau treffen lassen. Er wird Verlust erleiden in seinem Leben. Manchen wird keine Ehefrau Wert [zukommen] lassen." (pBerlin 8345, 3, 7-10). ${ }^{19}$ Allerdings sollte man betonen, dass derart ganz überwiegend negative Ankündigungen in den demotischen astrologischen Texten ungewöhnlich sind. Gelegentlich gibt es rein gute Schicksale, meist allerdings, und da zeigt sich durchaus lebenspraktische Realitätserfahrung, durchwachsene. Die Formulierungen sind in einer Weise gewählt, die zunächst den Eindruck erweckt, dass es sich um gesicherte, unumstössliche Fakten handelt.

Jedoch gibt es von anderer Seite her dann doch die Option, dass astral vorherbestimmtes Schicksal nicht als unabänderlich angesehen wurde. Wichtigstes Zeugnis hierfür ist ein griechisch überlieferter Text, ein komplexes Ritual, das PGM XIII überliefert ist. Es steht im Zusammenhang einer direkten Konsultation der Gottheit, die neben Fremdeinflüssen auch eine sehr erhebliche Menge ägyptischer Elemente zeigt und mutmasslich in Ägypten selbst in der Römerzeit entstanden ist. ${ }^{20}$ Dabei soll einem ein Engel erscheinen, den man um die Offenbarung des eigenen Horoskops bittet. Im Falle schlechter Auskünfte bittet man ihn, das Unheil und Verhängnis auszutilgen. Ein Gebet an den Weltenherrscher im gleichen Sinne schliesst sich an (PGM XIII 609-640). Im weiteren Verlauf der Handschrift kommt das Thema nochmals auf. In inhaltlich ganz gleichartiger Tendenz wird wieder die Auskunft über das Horoskop gesucht, im Falle schlechter Auskünfte aber nicht verzweifelt, sondern der Gott gebeten, es zu tilgen. Hier findet sich die explizite Angabe, der Gott vermöge alles (PGM XIII, 708-714). ${ }^{21}$ Auf derselben Linie liegt es auch, wenn bei Apuleius, Metamorphosen XI, 25 Lucius Isis u.a. im Hinblick darauf preist, dass sie die unheilvollen Bewegungen der Sterne unter Kontrolle halten würde. ${ }^{22}$

Zusammengenommen ergibt sich für die divinatorischen Techniken folgendes Bild: Die traditionell gewachsenen Techniken, insbesondere Traumbücher und terrestrische Omina, gehen grundsätzlich davon aus, dass Unheilsverkündigungen nicht definitiv sind. Einerseits wird teilweise schon mit der Ankündigung des Risikos die Verheissung der letztlichen Rettung verbunden, andererseits wird das Flehen zur Gottheit, gegebenenfalls durch Opfergaben unterstützt, als sinnvolles Verfahren empfohlen. Schicksal wirkt hier also nicht wie deterministische Gewissheit, sondern kann von den Göttern abgewendet werden. Neben dem sprachlichen Ausdruck als „Gnade“ (htp) ist dabei auch die Formulierung vom Reinigen, Abwischen oder Beseitigen des Bösen belegt.

18 Hierzu passt auch, dass nach Chairemon, überliefert bei Porphyrios, Epistula ad Anebonem II, 13 die meisten Ägypter ihr Schicksal von den Bewegungen der Sterne abhängig sehen, allerdings in dem Sinne, dass die von ihnen kultisch verehrten Gottheiten Löser vom Schicksal sein können, vgl. vaN DER Horst, Chaeremon, $14 \mathrm{f}$. (fr. 5) und 55.

19 Text in Photographie publiziert von Spiegelberg 1902, Taf. 97; vgl. Hughes 1986 sowie Quack 2008, 368-370.

20 Zum Text vgl. Merkelbach 1992.

21 Vgl. Gundel 1968, 68 u. 70f.; von Lieven 1999, 116.

22 Vgl. Griffiths 1975, 100f. u. 322; es geht hier sicher um Planetenpositionen. 
Die Astrologie als rezente Neuerfindung sieht sich mehr auf dem Boden eines absolut sicheren Fundaments unumstösslicher Regeln, was auch damit zusammenhängt, dass sie allein Aussagen über das ganze Leben gibt, während die anderen divinatorischen Techniken nur für die Aktualität mit begrenztem Zukunftsraum relevant sind. Sie operiert somit viel mehr in einer deterministischen Konzeption, was auch im Einklang mit den griechischsprachigen astrologischen Traktaten steht. ${ }^{23}$ In den Händen der konkreten Nutzer scheint diese Wertung aber keineswegs die einzig anerkannte gewesen zu sein; vielmehr hat man im Rahmen des traditionellen Weltbildes, in dem die Götter über dem Schicksal stehen, auch bei der Astrologie die eigentlich im System nicht vorgesehene Option entwickelt, schlechte Konstellationen zu ändern.

Als zweites Gebiet möchte ich die Lebenslehren aufgreifen. Aus der Spätzeit gibt es hier einige sehr umfangreiche Kompositionen. ${ }^{24}$ Besonders wichtig ist das grosse demotische Weisheitsbuch, das meist nach der besterhaltenen Handschrift als Papyrus Insinger bekannt ist. Der Text ist in 25 durchnumerierte Maximen strukturiert, die ein durch den jeweiligen Titel vorgegebenes Thema umreissen. Die Maximen an sich bestehen mehr aus Aussagesätzen, die Erfahrungen widerspiegeln, als aus direkten Anweisungen. Aber auch diese Aussagesätze sind natürlich in der Realität leicht in Folgerungen für das eigene Verhalten umzumünzen.

In dieser Lehre gibt es ein sehr ausgefeiltes Konzept von Vergeltung. ${ }^{25} \mathrm{Ihm}$ ist eine ganze Maxime gewidmet, und zwar konkret die 25. und letzte; dieser Komplex erhält durch die Positionierung eine herausragende Relevanz innerhalb des Werkes. Betitelt ist der Abschnitt als „Die Art, dich vor der Vergeltung zu hüten, damit kein Teil von ihr dich erreicht“. Darin wird ausgemalt, wie alle Missetaten eine angemessene Vergeltung finden. Am prägnantesten kann man es wohl mit einem Zitat dreier Zeilen fassen: „Die Vergeltung hört nicht auf, dem Kummer zu bereiten, der böse war. Geschick, Segen und Autorität hängen von ihrer Stimme ab. Sie vollzieht die Strafe für Vergehen, sie belohnt Wohltaten.“ (34, 17-19).

Jedoch ist die Vergeltung offenbar nicht alleinige Agency, die ohne göttliche Beteiligung im Rahmen eines schlichten Tun-Ergehen-Zusammenhanges handeln kann; vielmehr ist auch der Gott selbst involviert. Die Zusammenarbeit der beiden Grössen sieht man in „Der Gott vergisst nicht, die Vergeltung rastet nicht. $(33,10)$ “ oder in „Man kann den Gott und die Vergeltung nicht vermeiden in dem, was sie einem bestimmt haben“ $(34,21)$. In der letzten relevanten Passage „Jeder, der auf Schmerz entbrannt ist, den wird der Gott schmerzlich verbrennen." $(34,22)$ erscheint sogar allein die Gottheit als Vollstrecker.

Zunächst wirkt das Vorgehen fast nach einem Automatismus, auch hier ist aber der Komplex einer göttlichen Gnade, die über dem Schicksal steht, zu fassen. Am deutlichsten erscheint dies in: „Sorge und Kummer gibt es nicht zur Zeit der Gnade des Gottes“ $(34,16)$.

Ebenso aufschlussreich ist die hieran anschliessende letzte Passage. Dort formuliert der Autor oder der Schreiber der konkreten Handschrift im Stil eines negativen Sündenbekenntnisses, er habe nicht wissentlich etwas Schlechtes begangen. ${ }^{26}$ Darauf folgt dann: „Das Vergehen, das ich unwissentlich begangen habe, dafür bitte ich um Vergebung. Ich habe zum Gott

23 Vgl. hierzu Komorowska 2004, 281-334.

24 Vgl. den Überblick in Quack 2009, 111-146; Übersetzungen in HoffManN, Quack 2007, 230-304 u. $360-370$.

25 Der ägyptische Begriff ist $p$ 3 $\breve{c} b$ 3, was griechischem Nemesis entspricht, die wiederum im römerzeitlichen Ägypten beachtliche kultische Aufmerksamkeit erfuhr, vgl. Liсноска 2004. Für Vergeltungsideen in der demotischen Literatur vgl. QUACK 2015.

26 Die Behauptung von Stadler 2001, 188, ich hätte diese Passage in Quack 1999 nicht beachtet, ist faktisch falsch, s. QUACK 1999, 35. 
gerufen, dass er mir gnädig sei und mir eine Seefahrt ${ }^{27}$ ohne Verhör gewähre.“ $(35,4)$. Diese Sektion, die eindeutig die Themen der direkt vorangehenden Maxime über die Vergeltung aufgreift, zeigt also nochmals eindeutig, wie auch im Rahmen der Mechanismen der Vergeltung Raum für göttliche Gnade ist.

Neben der speziellen Sektion über die Vergeltung spielt auch der Begriff des Schicksals eine erhebliche Rolle im Text. An bestimmten Objekten oder Aktionen haftet ein Schicksal (3, 24; 4, 12), und das Schicksal kann dem Menschen etwas zukommen lassen (4, 7; 7, 14; 15, 21; 18, 4. 6; 21, 4). Die meisten Formulierungen würden aus sich heraus auf eine autonome Rolle des Schicksals als eigenständiger Instanz hinweisen. Ausnahmsweise aber merkt man doch, dass es nicht das Schicksal allein ist, sondern die Gottheit eine Rolle spielt, z.B. „Das Schicksal und der Gott bringen Wohltat noch spätabends." $(19,15)$. Ebenso gibt es im direkten Zusammenhang mit der Erwähnung göttlicher Gnade auch die Formulierung „Er erzeugt Wohltat und Schicksal im reifen Alter." $(19,20)$.

An sich zeigt die Tatsache der Lehre als solche, dass man mit verstehbaren regelhaften Strukturen der Welt rechnet. Jedoch ist es gerade für diesen Text besonders auffällig, wie er es nicht dabei belässt. Vielmehr gibt es am Ende jeder Maxime, ausgenommen der letzten, einen „paradoxen“ Schluss, welcher anzeigt, wie es auch dazu kommen kann, dass genau das Gegenteil der normalen Ergebnisse von Handlungen eintritt. ${ }^{28}$ Ein Beispiel ist etwa:
„Mancher lebt von wenig, um zu sparen, und wird doch arm.
Mancher kann nichts und das Schicksal gibt Reichtümer.
Es ist nicht (notwendigerweise) der sparsame Weise, der ein Vermögen(?) findet.
Es ist auch nicht (notwendigerweise) der Verschwender, dem Armut zuteil wird.
Der Gott gibt Reichtümer im Überfluss ohne Einkommen.
Aber er erzeugt auch Armut in der Börse ohne Ausgaben." (pInsinger 7, 13-18).

Schon die Ägypter selbst scheinen derartige Aussagen nicht immer ganz unproblematisch gefunden zu haben. Dies zeigt sich besonders daran, wie eine fragmentarische Parallelhandschrift, die eine stark überarbeitete Fassung des Werks bietet, die Paradoxe oft entschärft und besonders die schockierendsten Passagen, nämlich diejenigen über das mögliche schlechte Geschick derjenigen, die sich eigentlich konform zur Lehre verhalten haben, öfters streicht. ${ }^{29}$

Erhebliche Diskussion erfahren hat eine Passage, die refrainhaft am Ende jeder der einzelnen Lehren des Textes, also im direkten Anschluss an die paradoxen Schlüsse steht. ${ }^{30}$ Es gibt zwei leicht verschiedene Varianten davon. Die erste lautet $p 3$ šy irm p3 shne nti ly i p 3 nčr p 3 nti

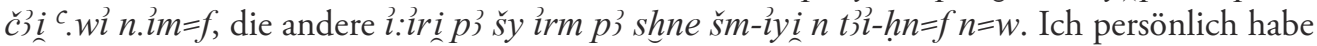
hierfür die Übersetzung: „Das Schicksal und das Geschick, das kommt - es ist der Gott, der es leitet“ bzw. als Variante „Schicksal und Geschick kommen und gehen in dem, was er (Gott) ihnen befohlen hat." befürwortet. ${ }^{31}$ Die Schlüsselbegriffe, auf die es ankommt, sind $\check{s}(3) y$ und

27 Die Seefahrt bezieht sich auf eine rituelle Bootsfahrt beim Totengericht, s. QuACK 1999. Der Versuch von STADLER 2001, 189-196, stattdessen „Freund“ zu lesen und dies auf Anubis als Totenrichter zu beziehen (übernommen auch von SмIтн 2007, 26f.), ist paläographisch ausgeschlossen. Für eine detaillierte Diskussion s. Quack 2014/15.

28 Vgl. Liснтнеiм 1983, 138-150.

29 Vgl. QuACK 2009, $115 \mathrm{f}$.

30 Vgl. Thissen 2002/2003, 98-102; ihm folgend Stadler 2012, 91.

31 QUACK 2009, 116. 
shne. Ersteres ist unproblematisch als normales ägyptisches Wort für „Schicksal“, und zwar aus sich heraus nicht eindeutig auf positive oder negative Markierung festgelegt, sondern neutral. ${ }^{32}$

Heikler ist dagegen der zweite Begriff. Bilinguen und Quasi-Bilinguen stellen sicher, dass das Substantiv shn primär griechischem $\tau u ́ \chi \eta$ entspricht, ${ }^{33}$ das dazugehörige Verb griechischem $\sigma v \mu \beta \alpha i ́ v \omega$,sich ereignen, eintreffen“. Speziell im Plural wird $n 3$ shn.w in demotischen Omentexten in den Sektionstiteln verwendet, die etwa "die Vorzeichen des Tieres x“, oder „die Vorzeichen des Sternes y“ lauten. ${ }^{34}$

Das Problem hierbei ist natürlich die Mehrdeutigkeit des griechischen $\tau u ́ \chi \eta$. Es bedeutet den Wörterbüchern zufolge sowohl „Zufall“ als auch „Geschick“, und es kann sowohl für den Unglücks- als auch den Glücksfall stehen. Thissen möchte im Papyrus Insinger shn im Sinne von „Zufall“ übersetzen. Mir erscheint es allerdings nicht recht nachvollziehbar, wie etwas von Gott Bestimmtes als „Zufall“ bezeichnet werden kann. Hinzu kommt, dass eben das Wort shn auch die Vorzeichen bezeichnet, welche in Omentexten kasuistisch aufgelistet werden. Im grossen demotischen Weisheitsbuch selbst wird an einer Stelle vom Vorzeichen der Himmelskonstellationen gesprochen und eben das Wort shne verwendet, wo eine Übersetzung als "Zufall“ dem Text keinesfalls gerecht würde (pInsinger 32, 5). Ferner ist auf der syntaktischen Ebene zu beachten, dass in der ersten der beiden Versionen des Refrains die beiden Begriffe durch ein singularisches Suffix aufgenommen werden, also mehr als ein Hendiadyoin denn als zwei wirklich verschiedene Begriffe verstanden werden. Letztlich sehe ich in $\check{S} y$ mehr das konkrete Einzelschicksal, in shne dagegen die Zukunftsverkündigung als System.

Unabhängig von den Detailfragen der Auffassung kann aber in einem grundlegenden Punkte Einigkeit bestehen. Der Gott und seine Anweisungen stehen oberhalb des Schicksals, und damit haben wir eine Konstellation, welche sich nicht merklich von derjenigen der divinatorischen Traktate unterscheidet.

Auch die weiteren demotischen Lebenslehren dürften in dieselbe Richtung gehen. In der Lehre des Chascheschonqi, die mehr aus isolierten Sprüchen besteht, spielen Schicksalskonzeptionen keine gleich grosse Rolle. Es gibt allerdings eine Passage, welche an die Paradoxe des grossen demotischen Weisheitsbuches erinnert. Sie lautet:

„[Manch ... .] ist bitter zu trinken.

[Manch ... ist geeignet,] einen Mann hinauszuwerfen.

[Manch] Stock ist geeignet, ihn herbeizuholen.

Manche Haft ist geeignet, am Leben zu erhalten.

Manche Freilassung ist geeignet zu töten.

Mancher spart, ohne (es wieder)zufinden.

Sie alle sind in der Hand des Schicksals, des Gottes." (26, $x+2-8)$

32 Vgl. Quaegebeur 1975.

33 Quaegebeur 1975, $141 \mathrm{f}$.

34 Angesichts der Verwendung auch in Tierominatexten (und in älterer Zeit etwa in dem von Vernus 1981 veröffentlichten Text) erweist sich die von Hughes 1986, 58 postulierte Äquivalenz zu griechischem $\tau \dot{\alpha}$

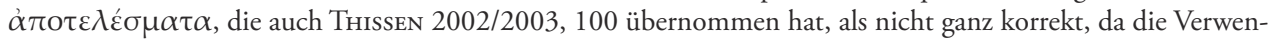
dung dieses Ausdruckes für Vorzeichen im griechischen Bereich auf astrologische Lehren beschränkt ist. Vgl. für die ältere Zeit ENMARCH 2008, $117 f$. 
Dabei erlauben es die Gegebenheiten der demotischen Sprache für den letzten Spruch, der übrigens fast textgleich auch pInsinger 30, 15 steht, sowohl eine Apposition „das Schicksal, nämlich der Gott“, als auch eine Aufzählung „das Schicksal und der Gott“ zu verstehen. Im Rahmen des pInsinger, wo man in den Refrains das Schicksal explizit als eine dem Gott untergeordnete Grösse präsentiert, ist letztere Deutung die plausiblere.

Auch ein relativ schlecht erhaltener Papyrus im Brooklyn Museum mit einem Weisheitstext ${ }^{35}$ bezeugt, dass das Schicksal eine Verbindung mit der Vergeltung von Unrechtstaten besitzt: „Das Schicksal findet seine Hand an ihm, um es ihm zu vergelten. Es gibt keinen, der sich vor ihm retten könnte.“ (3, 4-5).

Zusammengenommen erwecken viele Passagen in den demotischen Weisheitslehren aus sich heraus zunächst den Anschein, als sei das Schicksal hier eine autonome, nicht hintergehbare Grösse. Genaue Lektüre des Gesamttextes zeigt aber, dass die Gottheit immer die Oberhand hat und eingreifen kann, auch wenn man ein direktes Eingreifen, besonders im Sinne einer Abänderung normaler Konsequenzen, wohl als Ausnahme betrachtet hat.

Zum Abschluss seien einige strikter religiöse Kompositionen vorgestellt. Relevant ist zunächst eine Passage, die sich im Ritual zum Eintritt in die Kammer der Finsternis findet, das in seiner ersten Publikation eher unglücklich als Thotbuch bezeichnet wurde. ${ }^{36}$ Darin berichtet der Kandidat, was er im Rahmen einer Reise durch arkane Regionen erlebt hat:

\footnotetext{
„Ich nahm eine Lampe in meine Hand, um das Innere des Platzes vor ihr(?) zu erkennen. Ich gelangte in sein Inneres, ich erreichte einen Speicher des Lebens, der schwamm(?), ohne dass er sich auflöste(?).

Ich fand sechs Ruderer, die dasassen, indem sie vereint waren, indem sie in einer Redeweise priesen,

indem sie die Herzen vereinten mit denen von Ober- und Unterägypten zu den Ländern, welches ihre Herrin ist.

Siehe, sie haben keinen Feind, sie verbergen sich nicht, während sie eine krankhafte Fundierung niedertreten(?),

während sie einen Leidenden heilen, für den es kein Buch gibt, indem sie Sünden mit ihren Aussprüchen abwischen, indem sie einen Mann vor seinem Schicksal retten, während sein Tod hinter ihm dasteht." (B 04, 8/6-14).
}

Auch hier gibt es somit die Vorstellung, welche wir schon bei der Behandlung der divinatorischen Texte bemerkt hatten: Die göttlichen Wesen stehen über dem Schicksal und können es intentional verändern. In der konkreten Passage scheint die Frage von Sünden, die ein Mensch begangen hat, im Zentrum zu stehen und als Reaktion darauf eine an sich tödliche Krankheit erfolgt zu sein, für die in den medizinischen Bücher keine Behandlungsmethode zu finden ist. Auch sie kann durch dezidierte göttliche Eingriffe aber abgewendet werden.

35 Edition Jasnow 1992; neueste Übersetzung in Hoffmann, Quack 2007, 230-238 u. 360f.; vgl. weiter die Angaben in Quack 2009, 125-128.

36 Eine nicht wirklich befriedigende Textedition in JASNOw, ZAUZICH 2005; vgl. meine eigenen Bemerkungen in QUACK 2007a; ders. 2007b; ders., 2009, 160-168 (unter Einschluss einiger Fragmente, die mir zum Zeitpunkt meiner früheren Aufsätze noch nicht bekannt waren); s. auch STAdLER 2012, 177-187. Neue englische Übersetzung JaSNOw, ZaUZICH 2015. 
Als wesentlicher religiöser Text einschlägig ist auch der sogenannte „Mythos vom Sonnenauge. " ${ }^{37}$ In ihm unterhalten sich ein Hundsaffe und eine Katze tiefsinnig über Gott und Welt, wobei die beiden theologisch konnotiert sind, nämlich die Katze als Hathor-Tefnut, Tochter des Sonnengottes, die sich von ihm entfernt hat und nach Nubien gezogen ist, der Hundsaffe als Sohn des Weisheitsgottes Thot. Das Schicksal spielt hier verschiedentlich eine Rolle. In der Mehrzahl der Fälle wird es in einer Art thematisiert, als sei es autonome Entscheidungsinstanz, ja folge sogar rein automatisiert-routinierten Vorgaben.

Ereignisse speziell auffälliger und überraschender Art werden besonders auf das Schicksal zurückgeführt, so in: „[. . .] du zürnst ihm wegen der Angelegenheit des kleinen harten Knochens. Er [erschien(?)] im Sonnenlicht, bevor das Schicksal mich ihm begegnen liess. " (Lille A 19); oder „und ich würde sagen: „Siehe, meine Speise, die brachte mir das Schicksal zu Füssen, ich bin ihr nicht nachgegangen." (Lille A 26).

Ein spezieller Zuständigkeitsbereich des Schicksals ist dabei auch die Vergeltung, und hier wird der Punkt eindrücklich gemacht, dass niemand glauben sollte, stark genug zu sein, um vor ihr verschont zu bleiben. Vielmehr wird die Fähigkeit des Schicksals herausgestellt, immer einen noch stärkeren als Strafinstrument zu nutzen: „Die Re[ser]ve des Schicksals ist verborgen. Ein Weiser ist's, der sie kennt. Der Starke, es gibt einen Stärkeren als ihn. Der Mächtige, es gibt einen Mächtigeren als ihn. Der Bullige, es gibt einen Bulligeren als ihn. Der Kräftige, es gibt einen Kräftigeren selbst als ihn." (Leiden 16, 32-17, 7).

Dieser Passus sollte in Verbindung mit einer vorangehenden Diskussion im Text gelesen werden, in der es darum geht, wie jeder Mord gerächt wird - selbst in der Tierwelt: „Nichts auf Erden geschieht, ausser dem, was der Gott anordnen wird im Horizont. Wer eine gute Tat vollbringt, den sucht sie heim, und eine schlechte Tat ebenso." (Leiden, 14, 29-31); sowie „Wer tötet, den tötet man. Wer zu töten befiehlt, dessen Vernichtung [befiehlt] man. Ich habe die besagten Dinge gesagt, damit es dir verständlich wird, dass es keine [Angelegenheit] gibt, die vor dem Gott verborgen sein könnte. Re, das Licht, der Vergelter der Götter -Variante: Der Gott -, nimmt Rache für alles, was auf Erden ist, angefangen mit der Schmeiss(?)fliege, die das allergeringste Geschöpf ist, bis seine Strafe den Greifen erreicht hat, der wiederum das allergrösste Geschöpf auf Erden ist. D.h., die gute Tat und die böse Tat, die man auf Erden begehen wird, Re ist es, der es vergilt." (Leiden 15, 6-12). Dabei zeigen diese Textstellen bereits, dass die Gottheit selbst, speziell der Sonnengott, involviert ist und es sich nicht rein um einen abstrakten Tun-Ergehen-Zusammenhang handelt.

Auch sonst ist erkennbar, dass es sich doch nicht um eine rein automatische Maschinerie handelt. Bemerkenswert ist vielmehr, dass das Schicksal sich offenbar Emotionen erlaubt. Es liebt bestimmte Personen, und mit manchen erlaubt es sich auch einen Scherz. So heisst es: „»Mögest du auf deine Tenne fallen, mögest du deine Sykomore finden «, so spricht das Schicksal zu seinem Geliebten.“ (Leiden 5, 29-30). Oder auch: „Das Schicksal wollte sich einen Scherz erlauben wegen der dreisten Worte, die der Löwe gesagt hatte." (Leiden 18, 26-27).

Im Komplex der Emotionen zu sehen ist wohl auch eine andere, in ihrer Formulierung recht auffällige Aussage, nämlich: „Ich selbst bin hier, bis das Schicksal sich an mir gesättigt

37 Überblick in Quack 2009, 148-159; Übersetzung in Hoffmann, Quack 2007, 195-229 u. 356-360. Die beiden Haupthandschriften werden hier als „Lille“ bzw. „Leiden“ zitiert. Inzwischen hat sich eine internationale Forschergruppe konstituiert, die an einer vollständigen Neu- bzw. Erstedition der zahlreichen (zum größeren Teil noch unpublizierten) Handschriften arbeitet. 
hat." (Leiden A 12, 3-4). Sprecher ist dabei der kleine Hundsaffe, der sich in Nubien befindet, und im konkreten Zusammenhang geht es um die Frage der Rückkehr nach Ägypten. An sich möchte er nach Ägypten zurückkehren, hat damit aber offenbar Probleme. Leider sind die Zusammenhänge insofern undurchsichtig, als der Anfang des Textes bislang noch nicht rekonstruierbar ist und damit bestimmte Grundsituierungen unklar bleiben. Es wirkt aber so, als ob der Hundsaffe sich in dem Sinne dem Schicksal unterworfen sieht, dass es ihn vorerst dort festhält. Die „Sättigung“ impliziert offenbar, dass das Schicksal den Eindruck gewonnen hat, er sei genügend gestraft worden. ${ }^{38}$ Auch damit dürfte das Schicksal aber als subjektive, emotionsbehaftete Grösse erscheinen.

Zumindest jargonhaft kann sogar die nubische Katze als eine Gesprächspartnerin zur Ins$\operatorname{tanz}$ gemacht werden, welche das Schicksal lenkt. „Meine Herrin! Deine Schritte entscheiden [mein] Schicksal, Erhabene, millionen[fach(?).] Bei deiner Stärke! Wenn du mich vor dieser Strafe rettest und [mir] den Fehl [verzeihst(?)], werde ich meinerseits es [ver]gelten und dich aus deiner Gefahr erretten zu der Zeit, in der sie kommt." (Leiden 13, 15-19). Dabei gilt es aber festzuhalten, dass hier der Prozess in beide Richtungen laufen kann. Nicht nur hat die nubische Katze an dieser Stelle die reale Möglichkeit, den Hundsaffen entweder zu vernichten oder ungeschoren davon kommen zu lassen, sondern auch umgekehrt redet der Hundsaffe nicht nur davon, er könne es vergelten, sondern erweist sich im weiteren Verlauf der Handlung tatsächlich als jemand, der realiter die Göttin aus der Gefahr erretten und damit ihr Schicksal positiv beeinflussen kann.

Eine Schlüsselstelle hinsichtlich der jeweiligen Zuständigkeit von Gott und Schicksal ist eine der Erörterungen des Hundaffen: „Der Mensch mit seinem Schicksal ist wie ein Boot, das im Fahrtwind [segelt]. Sein Schicksal ist wie das sichere Ufer(?) [...] Bestattung. Der Wind zum Kentern(?), dessen Richtung, Farbe und [...] man nicht kennt, [...] Die Änderungen des Windes sind wie die Schicksalsschläge. Der Gott ist wie der Schiffer, der es steuert. Sein Herz ist sein Steuerruder [...] sein [...] wiederum. Wer wohltätig ist in seinem Erfolg, den lässt er an das sichere Ufer kommen. Wer aber grausam ist, der gleitet aus. Sein ... ist sein... [...] sofort. Der Gott ist der... der Fähre. Sein Werk ist, täglich zu leiten. Tägliche Leitung besorgt er. Derjenige, dem er zürnt, den wirft er hinaus; derjenige, dem er gnädig ist, den holt er herein." (Lille A 31-37). Solche Formulierungen zeigen m.E., dass eben das Schicksal nach spätägyptischer Konzeption keine rein autonome und automatisierte Grösse war, sondern die Gottheit stets eingreifen und der Sache einen anderen Kurs geben konnte. Dies mag auch eine übergreifende Warnung sein, nicht dort, wo scheinbar das Schicksal allein und autonom agiert, von einem Automatismus ohne Mitwirkung der Gottheit auszugehen.

Dem entspricht auch, was an einer anderen Stelle der Handschrift, in der Fabel von der Katze und der Geierin (Leiden 2, 6-3, 15) zur Sprache kommt. Dort geht es um Vergeltung. Sie kommt aber nicht ganz von selbst, sondern wird dadurch ausgelöst, dass die Katze ein Gebet an den Sonnengott richtet, und dieser den Vergeltungsdämon aussendet.

Handelt es sich hier um Vergeltung zu Lebzeiten, so sollte ein anderer Komplex zumindest kurz angesprochen werden, nämlich die Vergeltung nach dem Tod. ${ }^{39}$ Hierzu gibt es eine klare

38 Vgl. den Eigennamen $s y-p 3$ - $m w t$, der Tod hat sich gesättigt“ LüDdeckens, Thissen 1980-2000, 902. Er impliziert offenbar, dass der Tod durch Kindersterblichkeit schon so oft zugeschlagen hat, dass er jetzt satt sein und das Kind verschonen sollte.

39 Griffiths 1991, 201-242. 
Äusserung in einem noch unpublizierten Text, nämlich pWien D. 10014+10103a+b: „Jede Missetat, die ein Mensch auf Erden begeht - wenn er stirbt, vergilt man sie ihm.“

Schliesslich ist es angemessen, auf Isis als Herrin über das Schicksal einzugehen, womit wir auch den Brückenschlag von den ägyptischsprachigen Quellen zu den griechischen und lateinischen erbringen können. ${ }^{40}$ Eine Passage, welche Isis direkt als Schicksal auffasst, scheint es bereits in den demotischen Ostraka des Hor aus der mittleren Ptolemäerzeit zu geben. Die Details der betreffenden Stelle oHor 10, 8-9 bedürfen allerdings genauerer Diskussion. ${ }^{41}$ Konkret lautet der Text mit einigen für die aktuelle Argumentation nicht ausschlaggebenden Unsicherheiten, die besonders dadurch bedingt sind, dass ein Teilbereich über einem getilgten

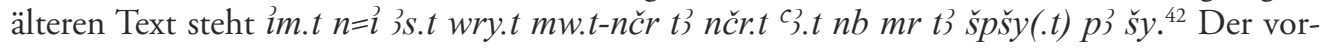
dere Teil lautet „Komm zu mir, Isis, Grosse, Gottesmutter, die grosse Göttin, die Herrin der Liebe!“. Problematisch ist lediglich, ob man die nachfolgenden Elemente mit Kockelmann als „Edle (Agathe Tyche), Geschick“ verstehen und direkt auf Isis beziehen kann, oder ob es sich hier um zwei nebeneinander stehende Gestalten handelt, bei denen Isis nur als „Edeldame“ bezeichnet wird, während „Schicksal“ sich auf eine andere Gestalt, mutmasslich Osiris, bezieht. ${ }^{43}$ Zwar kann Isis ohne weiteres als Herrin des Schicksals bezeichnet werden, was in hieroglyphischen Texten oft genug belegt ist, jedoch gibt es keine wirklich unproblematischen Belege dafür, dass sie direkt als "Schicksal“ erscheint. ${ }^{44} \mathrm{Zu}$ beachten ist dabei auch dass die beiden Bezeichnungen $\check{s} p \check{s} y$ und $\check{s} y$ sich in der demotischen astrologischen Terminologie auf zwei Häuser der Dodekatropos beziehen, die in griechischer Formulierung als Agathe Tyche und Agathos Daimon auftreten. ${ }^{45}$ Ein demotisches Graffito in Dakke (Dakke 1, Z. 4-5), das

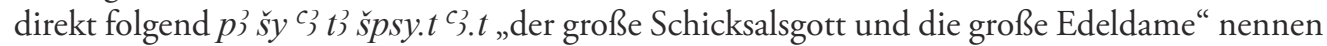
dürfte (die Lesung ist allerdings nicht völlig sicher) spricht wohl dafür, hier zwei nebeneinander stehende Gestalten anzusetzen. ${ }^{46}$

Potentiell relevanter ist der Papyrus Saqqara 2 rt., der auf jeden Fall von der Einmischung von Gottheiten, darunter der Isis, in menschliches Schicksal spricht, allerdings so schlecht erhalten und entsprechend heikel in seiner Interpretation ist, dass ich auf eine Heranziehung hier verzichten möchte. ${ }^{47}$ Dagegen von grösserem Interesse ist eine kurze demotische Erzählung aus den sogenannten „Krugtexten“, die vom Beistand der Isis berichtet und zeigt, wie ein armer Mann Leid und Unrecht erfährt, aber nach einem flehenden Anrufen von der Göttin Isis errettet wird, die dafür sorgt, dass er einen Schatz finden kann. ${ }^{48}$ Allerdings fällt hier nirgends direkt das Wort Schicksal, aber zumindest wird sehr deutlich, wie wichtig es nach ägyptischem Verständnis ist, die Gottheit anzuflehen.

40 Zu diesen s. Bergman 1967; Alvar 2008, $28 f$.

41 Vgl. Kockelmann 2008, 12f. u. 66.

42 Das anschliessende Element, das Kockelmann als ${ }^{3} 3$ liest und noch mit zu diesem Satz zieht, kann m.E. paläographisch aufgrund der Detailabweichungen im Vergleich mit sicheren Fällen von ${ }^{C}$ im Text nur als $k t$, anderes“ gelesen werden.

43 Fast dieselbe Formulierung (und dasselbe Problem) zeigt sich auch im demotischen Graffito Philae 408, Z. 5, s. Griffith 1937, 111-112.

44 Vgl. die Diskussion in Quaegebeur 1975, 86-87.

45 VON LIEVEN 1999, $123 \mathrm{f}$.

46 Griffith 1937, 18 f.

47 Smith, Tait 1983, 70-109; vgl. Quack 2009, 90-92.

48 Vgl. Hoffmann, Quack 2007, 178-180 u. 351; Quack 2009, $171 \mathrm{f}$. 
Abschliessend kann man feststellen, dass für die Alten Ägypter somit nicht das Schicksal, sondern die Gottheit und ihr Wille, einschliesslich der Bereitschaft zur Gnade, ${ }^{49}$ die oberste Instanz war. Dies äussert sich auch sehr markant in einer mehrfach mit nur geringen Varianten in demotischen Texten zu findenden Aussage „Es geschieht nichts, ausser dem, was der Gott befehlen wird“. ${ }^{50}$ In genau dieselbe Richtung geht es, wenn als letzter Satz der Lehre des Chascheschonqi formuliert wird: „Werde nicht müde, den Gott anzuflehen; er hat seine Stunde zu hören!“" $(28, \mathrm{x}+10) .{ }^{51}$

Joachim Friedrich Quack

Universität Heidelberg

Ägyptologisches Institut

Marstallhof 4

D-69117 Heidelberg

joachim_friedrich.quack@urz.uni-heidelberg.de

\section{Bibliographie}

Alvar 2008

J. Alvar, Romanising Oriental Gods. Myth, Salvation and Ethics in the Cults of Cybele, Isis and Mithras, Leiden-Boston 2008.

Assmann 1996

J. Assmann, Ägypten. Eine Sinngeschichte, MünchenWien 1996.

Assmann 1999

J. Assmann, «Kalendarische und messianische Geschichte, Altägyptische Formen geschichtlicher Semiotik», in H.D. Kittsteiner (Hg.), Geschichtszeichen, Köln-Weimar-Wien 1999, 15-30.

Assmann 2005

J. Assmann, «Zeitkonstruktion, Vergangenheitsbewusstsein und Geschichtsbewusstsein im alten Ägypten", in J. Assmann, K.E. Müller (Hgg.), Der Ursprung der Geschichte. Archaische Kulturen, das Alte Ägypten und das Frühe Griechenland, Stuttgart 2005, 112-214.

Aufrère, Mazoyer 2009

S.H. Aufrère, M. Mazoyer, (éds.), Clémence et Châtiment. Actes du Colloque organisé par le Cahiers Kubaba et l'Institut catholique de Paris 7-8 décembre 2006, Paris 2009.

\section{BERGMAN 1967}

Bergman, J., "I Overcome Fate, Fate Harkens to Me”. Some Observations on Isis as a Goddess of Fate», in H. RingGren (ed.), Fatalistic Beliefs in Religion, Folklore, and Literature. Papers read at the Symposium on Fatalistic Beliefs held at Åbo on the 7th-9th of September, 1964, Stockholm 1967, 35-51

Bouché-LeClercQ 1879

A.Bouché-Leclercq, Histoire de la divination dans l'antiquité. Tome I, Paris 1879.

Devauchelle 2008

D. Devauchelle, Rezension zu Stadler 2004, Die Welt des Orients 38 (2008), 242-245.

Dieleman 2009

J. Dieleman, Rezension zu Stadler 2004, Bibliotheca Orientalis 66 (2009), 225-231.

ENMARCH 2008

R. Enmarch, A world Upturned. Commentary on and Analysis of The Dialogue of Ipuwer and the Lord of All, Oxford 2008.

Gestermann 2008

L. Gestermann, "Zorn und Gnade ägyptischer Götter», in R.G. Kratz, H. Spieckermann (Hgg.), Divine

49 Vgl. zu diesem Komplex zusammenfassend Gestermann 2008; Sternberg-El Hotabi, 2008. Übergreifend s. auch Aufrère, Mazoyer 2009 (non vidi).

50 Vgl. Thissen 2002/2003, 101 für die Einzelbelege; pSaqqara I rt, fr. 3, 9, 25 ist allerdings die Deutung von mtw als Schreibung für $n t i-i l w$ angesichts des nicht determinierten vorangehenden $m t . t$ nicht möglich; ich sehe darin einen Konjunktiv (als Ersatz des Umstandssatzes des Futur III).

51 Vgl. Zauzich 1996 für die korrekte Deutung der Stelle sowie Quack 2009, 136 für das Aufgreifen dieser Formulierung in einem Gebet. 
Wrath and Divine Mercy in the World of Antiquity, Tübingen 2008, 19-43.

Griffith 1937

F. Ll. Griffith, Catalogue of the Demotic Graffiti of the Dodecaschoenus, Oxford 1937.

Griffiths 1975

J.G. Griffiths, Apuleius of Madauros. The Isis-Book (Metamorphoses, Book XI), Leiden.

GrIfFiths 1991

J.G. Griffiths, The Divine Verdict. A Study of Divine Judgement in the Ancient Religions, Leiden, New York, Kopenhagen, Köln 1991.

Gundel 1968

H.G. Gundel, Weltbild und Astrologie in den griechischen Zauberpapyri, München 1968.

Hoffmann, Quack 2007

F. Hoffmann, J.F. Quack, Anthologie der demotischen Literatur, Berlin 2007.

VAN DER HORST 1987

P.W. van der Horst, Chaeremon. Egyptian Priest and Stoic Philosopher. The fragments collected and translated with explanatory notes, Leiden-New York-Kopenhagen-Köln ${ }^{2} 1987$.

Hughes 1986

G.R. Hughes, "An Astrologers Handbook in Demotic Egyptian», in: L.H. Lesko (Ed.), Egyptological Studies in Honor of Richard A Parker Presented at the Occasion of His 78th Birthday December 10, 1983, Hanover/London 1986, 53-69.

Hughes 2005

G.R. Hughes, Catalogue of Demotic Texts in the Brooklyn Museum, Chicago 2005.

JASNOW 1992

R. Jasnow, A Late Period Hieratic Wisdom Text (P. Brooklyn 47.218.135), Chicago 1992.

JASNOW, ZAUZICH 2005

R. Jasnow, K.-Th. Zauzich, The Ancient Egyptian Book of Thot. A Demotic Discourse on Knowledge and Pendant to the Classical Hermetica, Wiesbaden 2005.

Kockelmann 2008

H. Kockelmann, Praising the Goddess. A Comparative and Annotated Re-Edition of Six Demotic Hymns and Praises Addressed to Isis, Berlin-New York 2008.

KomorowsKa 2004

J. Komorowska, Vettius Valens of Antioch. An Intellectual Monography, Krakau 2004.
LAISNEY 2010

V. Laisney, Rezension zu Stadler 2004, Orientalia 79 (2010), 411-415.

LICHOCKA 2004

B. Lichocka, Nemesis en Égypte romaine, Mainz 2004.

LichTHEIM 1983

M. Lichtheim, Late Egyptian Wisdom Literature in the International Context. A Study of Demotic Instructions, Freiburg/Göttingen 1983.

VON LIEVEN 1999

A. von Lieven, "Divination in Ägypten», Altorientalische Forschungen 26 (1999), 77-126.

LüDdeckens, Thissen 1980-2000

E. Lüddeckens, H.J. Thissen, Demotisches Namenbuch, Band I, Wiesbaden 1980-2000.

Maul 1994

St. Maul, Zukunftsbewältigung. Eine Untersuchung altorientalischen Denkens anhand der babylonisch-assyrischen Löserituale (Namburbi), Mainz 1994.

Merkelbach 1992

R. Merkelbach, Abrasax, Band 3. Zwei griechischägyptische Weihezeremonien. Die Leidener Weltschöpfung · Die Pschai-Aion-Liturgie, Opladen 1992.

Morenz, Müller

S. Morenz, D. Müller, Untersuchungen zur Rolle des Schicksals in der ägyptischen Religion, Berlin 1960.

NAETHER 2010

F. Naether, Die Sortes Astrampsychi. Problemlösungsstrategien durch Orakel im römischen Ägypten, Tübingen 2010 .

Prada 2012a

L. Prada, «Papyrus Berlin P. 8769: A New Look at the Text and the Reconstruction of a Lost Demotic Dream Book», in V. Lepper (Hg.), Forschungen in der Papyrussammlung. Eine Festgabe für das Neue Museum, Berlin 2012, 309-328.

PRADA 2012b

L. Prada, "Classifying dreams, classifying the world: ancient Egyptian oneiromancy and Demotic dream books», in H. Abd El Gawad, N. Andrews, M. Correas-Amador, V. Tamorri, J. Taylor (eds.), Current research in Egyptology 2011: proceedings of the twelfth annual symposium which took place at Durham University, United Kingdom, March 2011, Oxford/Oakville 2012, 167-177.

Prada 2013

L. Prada, "Dreams, bilingualism, and oneiromancy in Ptolemaic Egypt: remarks on a recent study", Zeitschrift für Papyrologie und Epigraphik 184 (2013), 85-101. 
QUACK 1999

J.F. Quack, «Balsamierung und Totengericht im Papyrus Insinger», Enchoria 25 (1999), 27-38.

QUACK 2005

J.F. Quack, Rezension zu Stadler 2004, Archiv für Papyrusforschung 51 (2005), 174-179.

QUACK 2006

J.F. Quack, "A Black Cat from the Right, and a Scarab on your Head. New Sources for Ancient Egyptian Divination», in: K. Szpakowska (Ed.), Through a Glass Darkly: Magic, Dreams, and Prophecy in Ancient Egypt, Swansea 2006, 175-187.

QUACK 2007a

J.F. Quack, «Die Initiation zum Schreiberberuf im Alten Ägypten», Studien zur Altägyptischen Kultur 36 (2007), 249-295.

QUACK 2007b

J.F. Quack, «Ein ägyptischer Dialog über die Schreibkunst und das arkane Wissen", Archiv für Religionsgeschichte 9 (2007), 259-294.

QUACK 2008

J.F. Quack, "Demotische magische und divinatorische Texte», in B. Janowski, G. Wilhelm (Hgg.), Texte aus der Umwelt des Alten Testaments, Neue Folge Band 4. Omina, Orakel, Rituale und Beschwörungen, Gütersloh 2008, 331-385.

QUACK 2009

J.F. Quack, Einführung in die altägyptische Literaturgeschichte III. Die demotische und gräko-ägyptische Literatur, 2. veränderte Auflage, Berlin 2009.

QuACK 2010

J.F. Quack, «Präzision in der Prognose, oder: Divination als Wissenschaft», in: A. Imhausen, T. Pommerening (Hgg.), Writings of Early Scholars in the Ancient Near East, Egypt, Rome, and Greece, Berlin/New York 2010, 69-91.

QuAck 2014/15

J.F. Quack, «Nochmals zu Balsamierung und Totengericht im großen demotischen Weisheitsbuch", Enchoria 34 (2014/2015), 105-118.

QUACK 2015

J.F. Quack, «„Sage nicht: »Der Frevler gegen Gott lebt heute«; auf das Ende sollst du achten!“. Gedanken der spätägyptischen Literatur zum Problem des Bösen in der Welt», in B. Ego, U. Mittmann (Hgg.), Evil and Death. Conceptions of the Human in Biblical, Early Jewish, Early Christian, Greco-Roman and Egyptian Literature (Berlin/New York 2015), 377-409.

Quaegebeur 1975

J. Quaegebeur, Le dieu égyptien Shaï dans la religion et l'onomastique, Leuven 1975.
Richter 2008

T.S. Richter, Rezension zu Stadler 2004, Wiener Zeitschrift für die Kunde des Morgenlandes 98 (2008), 380-386.

SMITH, TAIT 1983

H.S. Smith, W.J. Tait, Saqqâra Demotic Papyri I, London 1983.

SMITH

M. Smith, Traversing Eternity. Texts for the Afterlife from Ptolemaic and Roman Egypt, Oxford 2009.

SPIEGELBERg 1902

W. Spiegelberg, Demotische Papyrus aus den königlichen Museen zu Berlin, Leipzig 1902.

SpIEGELBERg 1932

W. Spiegelberg, Catalogue général des antiquités égyptiennes du Musée du Caire. Die demotischen Denkmäler III. Demotische Inschriften und Papyri (Fortsetzung) 50023-50165, Berlin 1932.

STADLER 2001

M.A. Stadler, "Zwei Bemerkungen zum Papyrus Insinger", Zeitschrift für Ägyptische Sprache und Altertumskunde 130 (2001), 186-196.

STADLER 2004

M.A. Stadler, Isis, das göttliche Kind und die Weltordnung. Neue religiöse Texte aus dem Fayum nach dem Papyrus Wien D. 12006 Recto, Wien 2004.

STADLER 2012

M.A. Stadler, Einführung in die ägyptische Religion ptolemäisch-römischer Zeit nach den demotischen religiösen Texten, Berlin 2012.

SternberG-El Hotabi 2008

H. Sternberg-El Hotabi, «Ich besiege das Schicksal«. Isis und das Schicksal in der ägyptischen Religion", in R.G. Kratz, H. Spieckermann (Hgg.), Vorsehung, Schicksal und göttliche Macht, Tübingen 2008, 40-60.

Thissen 2002/2003

H.J. Thissen, "Annotationes Demoticae», Enchoria 28 (2002/2003), 98-105.

VERnus

P. Vernus, «Omina calendériques et comptabilité d'offrandes sur une tablette hiératique de la XVIII ${ }^{\mathrm{e}}$ dynastie», Revue d'Égyptologie 33 (1981), 89-124.

Volten 1942

A. Volten, Demotische Traumdeutung (Pap. Carlsberg XIII und XIV Verso), Kopenhagen 1942.

WINKLER

A. Winkler, Rezension zu Stadler 2004, Lingua Aegyptia 15 (2007), 359-364. 


\section{ZAUZICH 1980}

K.-Th. Zauzich, "Aus zwei demotischen Traumbüchern", Archiv für Papyrusforschung und verwandte Gebiete 27 (1980), 91-98; Abb. 7-8.

\section{ZAUZICH 1996}

K.-Th. Zauzich, "Anchscheschonqi - eine Lehre für den Schreiber?», in M. Schade-Busch (Hg.), Wege öff- nen. Festschrift für Rolf Gundlach zum 65. Geburtstag, Wiesbaden 1996, 376-384.

\section{ZAUZICH 2012}

K.-Th. Zauzich, «Das demotische „Buch des Geckos“ und die Palmomantik des Melampous», in V. Lepper (Hg.), Forschungen in der Papyrussammlung. Eine Festgabe für das Neue Museum, Berlin 2012, 355-373. 